\title{
COMMERCIAL PRODUCTION OF HEAVY METAL FLUORIDE GLASS FIBER IN SPACE
}

\author{
Dennis S. Tucker \\ Space Sciences Laboratory \\ ES75 \\ Marshall Space Flight Center, Al 35812 \\ 205-544-2685
}

\author{
Gary L. Workman and Guy A. Smith \\ Center for Automation and Robotics \\ Universtiy of Alabama in Huntsville \\ Huntsville, Al 35899 \\ 205-890-6086
}

\begin{abstract}
International Space Station Alpha (ISSA) will provide a platform not only for materials research but also a possible means to produce products in space which cannot be easily produced on the ground. Some products may even be superior to those now produced in unit gravity due to the lack of gravity induced convection effects. Our research with ZrF4-BaF2-LaF3-AIF3-NaF (ZBLAN glass) has shown that gravity does indeed play a major role in the crystallization behavior of this material. At the present time ZBLAN is being produced on earth in fiber optic form for use in surgical lasers and fiber optic lasers among other applications. High attenuation coefficients, however, have kept this material from being used in other applications such as long haul data transmission links. The high attenuation coefficients are due to impurities which can be removed through improved processing techniques and crystals which can only be removed or prevented from forming by processing in a reduced gravity environment.
\end{abstract}

\section{INTRODUCTION}

Since the discovery of ZBLAN by Poulain (1975) a number of researchers have investigated heavy metal fluoride glass groups. Due to its resistance to crystallization ZBLAN has been one of the most heavily studied. This glass has the ability to transmit from the near ultraviolet to the mid infrared. It is the infrared transmitting capabilities which make this glass so attractive. It is being investigated as a fiber optic material, possibly as a replacement for fused silica in some instances. Applications include, nuclear radiation resistant links, IR lasers, remote sensing and ultralong repeaterless links (Tran et. al. 1982).

ZBLAN fiber optics for low-loss applications as a minimum should possess intrinsic attenuation levels in the 0.01 to $0.001 \mathrm{~dB} / \mathrm{km}$ range (Varma et. al. 1993). However, due to absorption from impurities and scatter due to crystallites and other inclusions, the theoretical intrinsic levels have not been attained to date. Absorption from impurities can be controlled by careful preparation of raw materials and fiber preforms. However, due to thermodynamic instabilities and propensity for crystallization in ZBLAN, microgravity processing may be the only method to rid the material of crystallites. 